\title{
Tratamiento de la hipersensibilidad dentinaria post terapia periodontal, mediante el uso de dos dentífricos desensibilizantes
}

\section{Dentin hypersensitivity treatment post periodontal therapy, using two desensitizing dentifrices}

\author{
Espinoza J'1, González L¹, Ruiz P²
}

\begin{abstract}
RESUMEN
Objetivo: El objetivo de este estudio fue evaluar y comparar la eficacia en la reducción de la hipersensibilidad dentinaria posterior a la terapia periodontal utilizando dentífricos que contienen arginina al $8 \%$ - carbonato de calcio versus acetato de estroncio al $8 \%$, tras una y tres semanas de uso de las pastas dentales. Materiales y Método: Estudio clínico, aleatorio, ciego y controlado con dos grupos paralelos, y tres semanas de seguimiento, en el cual el universo de trabajo fue de 20 pacientes con diagnóstico de periodontitis crónica generalizada leve o moderada y que hayan presentado hipersensibilidad dentinaria posterior a la terapia periodontal no quirúrgica en al menos un canino y/o premolar, y asociado a recesión gingival. Los pacientes fueron seleccionados aleatoriamente y se distribuyeron al azar en cada grupo de pastas dentales y fueron evaluados tras una y tres semanas de uso de los dentífricos. Se les aplicó aire proveniente de la jeringa triple del equipo dental en la zona cervical con hipersensibilidad, estandarizando la técnica. La cuantificación del dolor se realizó a través de la Escala Visual Análoga (EVA). Resultados: No hubo diferencia estadísticamente significativa entre el uso de los dentífricos que contienen arginina $8 \%$ - carbonato de calcio versus acetato de estroncio al $8 \%$ para la reducción de la hipersensibilidad dentinaria tras una y tres semanas de uso de las pastas dentales. Existió diferencia estadísticamente significativa en la reducción del dolor con el uso de ambos dentríficos a la primera y tercera semana de medición.
\end{abstract}

Rev. Clin. Periodoncia Implantol. Rehabil. Oral Vol. 6(2); 78-82, 2013.

Palabras clave: Terapia periodontal, hipersensibilidad dentinaria, arginina, carbonato de calcio, acetato de estroncio, desensibilizantes.

\section{ABSTRACT}

Aim: The aim of this clinical study was to evaluate and to compare the efficacy in reducing the dentin hypersensitivity after periodontal therapy using dentifrices which contain $8 \%$ arginine, calcium carbonate versus $8 \%$ strontium acetate, after one and three weeks of use of the dentifrices. Methods: A three-week clinical study with 20 subjects with diagnosis of slight to moderate chronic periodontitis with dental hypersensitivity after periodontal therapy, and presence of gingival recession in canines and/or premolars. Patients were randomly selected and assigned to each group and toothpastes were evaluated after one and three weeks of use. Air from a triple syringe was applied into the cervical area with hypersensitivity. The quantification of pain was performed using the Visual Analogue Scale (VAS). Results: There was not statistically significant difference between the use of the dentifrices which contain $8 \%$ arginine, calcium carbonate versus $8 \%$ strontium acetate in reducing dentin hypersensitivity after one and three weeks of use of the dentifrices. Nevertheless, there was a statistically significant difference in the reduction of pain using both dentifrices in the first and third week of measurement. Rev. Clin. Periodoncia Implantol. Rehabil. Oral Vol. 6(2); 78-82, 2013.

Key words: Periodontal therapy, dental hypersensitivity, arginine, calcium carbonate, strontium acetate, desenzitising.

\section{INTRODUCCIÓN}

La periodontitis es una patología de origen infeccioso que se caracteriza por la presencia de lesiones inflamatorias gingivales, que llevan a la formación de sacos periodontales, lo que lleva a la reabsorción de hueso alveolar y eventualmente a la pérdida del diente ${ }^{(1)}$

Para tratar la enfermedad periodontal existen dos modalidades de terapia: quirúrgica y no quirúrgica. Con respecto a esta última, consiste en realizar un destartraje supragingival, subgingival y/o pulido radicular para remover el cálculo dental y el biofilm ${ }^{(2)}$.

Como consecuencia del tratamiento y de la enfermedad periodontal en sí, los pacientes pueden presentar hipersensibilidad dentinaria, debido a que durante el pulido radicular se remueve el cemento acelular que se dispone normalmente en el área cervical inmediatamente adyacente a la superficie de la dentina radicular, induciendo la hipersensibilidad dentinaria ${ }^{(2,3)}$.

La hipersensibilidad dentinaria (HSD) se define como un dolor agudo, de corta duración, que se presenta cuando la dentina responde a un estímulo térmico, químico o físico, y que no se puede atribuir a ninguna otra patología ${ }^{(4-16)}$. Este es un problema relativamente común en la práctica del tratamiento periodontal. Puede que ocurra espontáneamente cuando la raíz ha sido expuesta como resultado de una recesión gingival y/o formación de sacos, después de realizado el pulido radicular y los procedimientos quirúrgicos ${ }^{(2)}$. Por otra parte, también puede deberse a erosiones del esmalte, ya sea por caries, traumatismos y/o dieta rica en ácidos ${ }^{(17)}$.

Hoy en día, este problema es muy habitual entre los pacientes periodontales, ya que se ha reportado una prevalencia considerablemente alta $(60 \%-98 \%)^{(18)}$. Con respecto a la distribución, los dientes que con mayor frecuencia se ven afectados son los caninos y premolares, y la región que se observa afectada generalmente es la zona cervical vestibular ${ }^{(15)}$

En la actualidad, aún se investiga el mecanismo exacto que provoca la hipersensibilidad dentinaria, pero la mayoría acepta la teoría hidrodinámica como una explicación a sus síntomas.

En relación a esta teoría, Brännstrom postuló que frente a un estímulo, se produce un movimiento del fluido contenido en los túbulos dentinarios, lo que provoca la deformación del odontoblasto. En e

1. Cirujano Dentista. Universidad Mayor. Chile.

2. Cirujano Dentista. Especialidad en Periodoncia e Implantología. Área de Periodoncia. Docente Universidad Mayor. Chile. 
odontoblasto y en su prolongación se produce la apertura de los canales iónicos de la membrana, entra $\mathrm{Na}+$ y se depolariza la fibra, generando un impulso eléctrico que origina el dolor. Los canales de sodio son activados por diferentes voltajes, ya sea por sustancias químicas o por presión mecánica. El calor expande el fluido intratubular que fluye hacia la pulpa y el frío lo contrae generando movimientos al exterior ${ }^{(18)}$.

La teoría hidrodinámica determina así que los estímulos que producen dolor aumentan el flujo centrífugo del contenido de los túbulos, provocando una modificación de presión en toda la dentina estimulando a las terminaciones nerviosas ${ }^{(18)}$.

Existen varios agentes desensibilizantes que han sido propuestos para el control de la hipersensibilidad dentinaria. Estos pueden ser de uso profesional y doméstico. Entre los más utilizados, se encuentran los dentríficos, que pueden contener flúor, además de ingredientes activos como cloruro de estroncio y nitrato de potasio(2). Por lo general, se utilizan como primera opción de tratamiento, ya que se ha observado que son efectivos, pero usualmente se demoran entre cuatro a ocho semanas en lograr una disminución del dolor ${ }^{(5)}$.

El mecanismo de acción más común es la reducción del diámetro de los túbulos dentinarios, mediante el uso de sales solubles que en contacto con el fluido dentinario, forman compuestos insolubles. De esta manera, se limita el desplazamiento del fluido dentro de ellos ${ }^{(2)}$

Por otra parte, existe otro mecanismo que consiste en interferir la transmisión dolorosa, a través del uso de las sales de potasio que provocarían la depolarización de las fibras nerviosas ${ }^{(19)}$.

Hoy en día existen nuevas líneas de dentrífricos, las que contienen arginina $8 \%$ - carbonato de calcio o acetato de estroncio al $8 \%$.

El producto desensibilizante Colgate Pro Alivio $\AA$, es un nuevo dentífrico que contiene arginina al $8.0 \%$, carbonato de calcio y 1.445 ppm de flúor. Establece que la interacción entre la arginina con el carbonato de calcio, provee de un ambiente alcalino que va a estimular el depósito de iones calcio y fosfato en la superficie dentinaria, obliterando los túbulos dentinarios abiertos ${ }^{(19)}$.

La arginina es un aminoácido, con carga positiva, a un $\mathrm{pH}$ fisiológico de 6.5 a 7.5 y que se obtiene de los alimentos o a través del ciclo de la urea. Poseería un efecto benéfico participando en la oclusión de los túbulos dentinarios abiertos ${ }^{(20)}$, resistente a la exposición de ácidos provenientes de la dieta, con propiedades de baja conductancia hidráulica y con una composición química baja en nitrógeno y carbono, pero alta en calcio, oxígeno y fósforo ${ }^{(20)}$.

El carbonato de calcio $\left(\mathrm{CaCO}_{3}\right)$ es un compuesto químico que se encuentra de forma abundante en la naturaleza. Es poco soluble, y en medios acuosos como el agua, puede disociarse en carbonato y calcio. La interacción entre la arginina y el carbonato de calcio participan en la obliteración de los túbulos dentinarios abiertos, resistentes a los ácidos provenientes de la dieta(20).

Por otra parte, existe otro agente desensibilizante que es el principal componente del dentífrico Sensodyne Rápido Alivio $®$, el acetato de estroncio. Se ha propuesto que este compuesto actúa depositando una fina capa de partículas insolubles, y también es absorbido a nivel del tejido conectivo de la dentina y del resto de los tejidos calcificados ${ }^{(7,21)}$. Todo este proceso produciría la obliteración de los túbulos dentinarios, con el consiguiente efecto desensibilizante ${ }^{(7)}$.

Este estudio tiene como propósito comparar la eficacia de dos dentríficos que contienen agentes desensibilizantes, Colgate Pro Alivio ${ }^{\circledR}$, cuyos agentes son la arginina al $8 \%$, carbonato de calcio y Sensodyne Rápido Alivio $₫$, cuyo componente desensibilizante es el acetato de estroncio al $8 \%$.

\section{MATERIAL Y MÉTODO}

Se desarrolló un ensayo clínico, aleatorio, ciego y controlado con dos grupos paralelos. El universo de trabajo fue de 20 pacientes de los alumnos de cuarto año de la asignatura de Periodoncia de la Universidad Mayor.

\section{Selección de Muestra}

Se seleccionó un grupo de 20 pacientes que se encontraban en tratamiento periodontal con alumnos de cuarto año de la Facultad de Odontología de la Universidad Mayor. Todos los pacientes que ingresaron al estudio de manera voluntaria, firmaron previamente un consentimiento informado, en el cual se explicó la naturaleza del trabajo de investigación y que podían optar la opción de abandonar el estudio.

El estudio contó con la aprobación de comité de ética de la Facultad de Odontología de la Universidad Mayor.

Los pacientes recibieron tratamiento periodontal convencional no quirúrgico, consistente en: instrucción en técnica de higiene oral, destartraje supragingival, subgingival y pulido radicular realizado por los alumnos de IV año de la carrera de Odontología de la Universidad Mayor. Además, debieron cumplir con todos los criterios de inclusión y exclusión establecidos. Entre los criterios de inclusión se requería ser voluntarios mayores de 18 años; diagnosticados con periodontitis crónica generalizada leve o moderada; con diagnóstico de hipersensibilidad dentinaria posterior al tratamiento periodontal no quirúrgico; poseer al menos un premolar y/o canino con hipersensibilidad, y asociado a recesión gingival; ser sistémicamente sanos o si presentan alguna enfermedad metabólica, ésta debía estar controlada.

Dentro de los criterios de exclusión se debían descartar a pacientes embarazadas; pacientes que habían recibido cualquier tipo de tratamiento para la hipersensibilidad dentinaria previo a la terapia periodontal; que se habían realizado blanqueamiento dental y/o fluoración; que presentaron piezas dentarias con signos de trauma oclusal, identificación de caries, fisuras de esmalte y/o dentina, ranura palatogingival y otros tipos de invaginaciones del esmalte, restauraciones recientes y/o fracturadas; con alguna reacción alérgica al dentífrico Colgate Pro Alivio ${ }^{\circledR}$ y/o Sensodyne Rápido Alivio ${ }^{\circ}$ o a alguno de sus componentes; que presentaron algún desorden alimenticio como bulimia o anorexia; o consumidores de una ingesta excesiva de ácidos; reporte de reflujo gastroesofágico; fumadores; que estuvieron bajo tratamiento crónico con Aines.

A cada paciente luego de una semana de efectuado el pulido radicular, se le preguntó si presentaba dolor agudo, de corta duración y si cedía después de aplicar un estímulo evaporativo proveniente de la jeringa triple. Aquellos que respondieron que no, se excluían inmediatamente de la muestra de pacientes. Los que respondieron que sí, se procedió a examinar la o las piezas dentarias afectadas y además se les pidió a los pacientes que describieran el tipo de dolor que presentaban para distinguir si correspondía al dolor característico de la HSD.

$\mathrm{Si}$ algún sujeto no cumplía con alguno de los criterios de inclusión, se excluían de la muestra de pacientes. Si éstos cumplían todos los criterios, se procedió a examinar los posibles diagnósticos diferenciales para confirmar el diagnóstico de HSD.

\section{Técnica para Evaluar la Hipersensibilidad Dentinaria}

Se aisló de forma relativa la pieza dentaria afectada con tórulas de algodón y se aplicó aire proveniente de la jeringa triple del equipo dental Adec® 80 psi, a una distancia de un centímetro (medido con la sonda periodontal) en forma perpendicular a la superficie cervical vestibular de la pieza dentaria durante cinco segundos y se procedió a preguntarle al paciente su dolor inicial (Figuras 1 y 2 ).

La cuantificación del dolor se realizó a través de la Escala Visual Análoga (EVA), utilizando un código numérico del 0 al 10, siendo 0 : ausencia de dolor y 10: el máximo de dolor (Figura 3). Por último, se registró el EVA inicial junto con la fecha en la ficha clínica de cada paciente.

\section{Indicaciones del Fabricante}

Sensodyne Rápido Alivio ${ }^{\circledR}$ (acetato de estroncio al 8\%): el paciente debe cepillarse sus dientes dos veces al día con la pasta dental Sensodyne Rápido Alivio. Una vez al día junto con el cepillado, el paciente debe aplicarse una pequeña porción de pasta directamente en el o los dientes sensibles con la yema del dedo y masajear durante 1 minuto. Posteriormente el paciente debe enjuagarse.

Colgate Pro Alivio ${ }^{\circledR}$ (arginina 8\% - carbonato de calcio): el paciente debe aplicar la pasta a un cepillo con cerdas suaves asegurándose de cepillar todas las áreas sensibles del diente. Se debe cepillar dos veces al día. Posteriormente, se debe enjuagar después del cepillado. Una vez dadas las indicaciones, se examinó a los pacientes tras una y tres semanas desde la medición del EVA inicial y se anotó la cuantificación del dolor a través de la Escala Visual Análoga, utilizando el método explicado anteriormente. 
Al ser un ensayo clínico controlado ciego, el operador $\mathrm{N}^{0} 1$ entregó las pastas dentales a los pacientes, por lo que sólo él conoció la marca del dentífrico y además les explicó la técnica de cepillado. Por otra parte, el operador №2 examinó a los pacientes, determinó el diagnóstico de HSD y desconocía la marca de pasta dental entregada a cada paciente.

\section{Diseño Experimental}

Los 20 pacientes fueron seleccionados aleatoriamente, los que se distribuyeron al azar en dos grupos de 10 pacientes a través del sistema Microsoft Office Excel.

De acuerdo al grupo que fueron asignados, el operador $\mathrm{N}^{01}$ realizó la entrega de tres tubos de pastas dentales para cada paciente que fueron donados por la empresa Colgate-Palmolive $\AA^{\circledR}$ y Sensodyne ${ }^{\circledR}$, y además de un cepillo dental de filamentos suaves donados por la empresa Sensodyne ${ }^{\circledR}$. Posteriormente, se realizaron las siguientes demostraciones a los pacientes para la utilización de los dentríficos:

Grupo A: se aplicó la pasta dental Sensodyne Rápido Alivio® sobre la superficie vestibular de la pieza dentaria afectada con la yema del dedo durante un minuto.

Grupo B: se aplicó la pasta Colgate Pro Alivio $®$ del tamaño de una arveja en un cepillo suave sobre la superficie vestibular durante un minuto.

Tanto en el grupo A y B se les entregó a cada paciente el agente terapéutico para uso doméstico, otorgándole un instructivo para su aplicación, según indicación del fabricante.

\section{Análisis Estadístico}

El análisis estadístico comenzó con la descripción de las variables evaluadas. La sensibilidad fue medida como variable ordinal.

Los resultados se analizaron mediante el test $\mathrm{t}$ no pareado. Se comparó el EVA inicial, y a la primera y tercera semana de uso de los dentríficos (Tabla 1). Se consideró que existían diferencias significativas si $p<0.05$. Asimismo, se analizó mediante regresión lineal el cambio de dolor entre el EVA inicial, y a la primera y tercera semana de tratamiento (Tabla 2). Los resultados fueron analizados a través del software Systat versión 13.1.

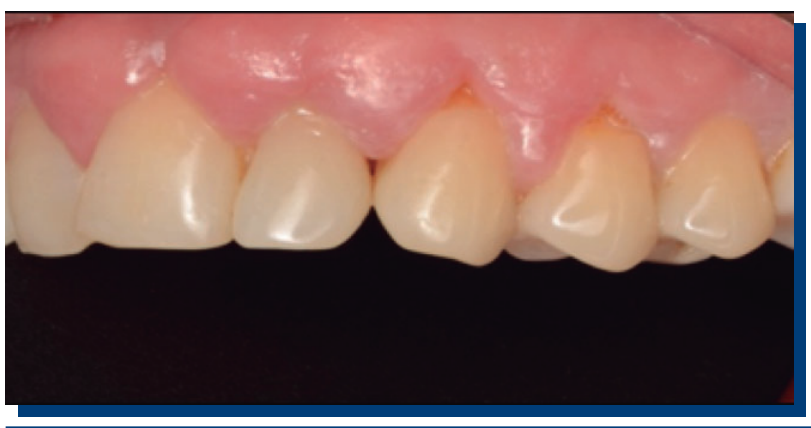

Figura 1. Pieza 11 con recesión gingival.

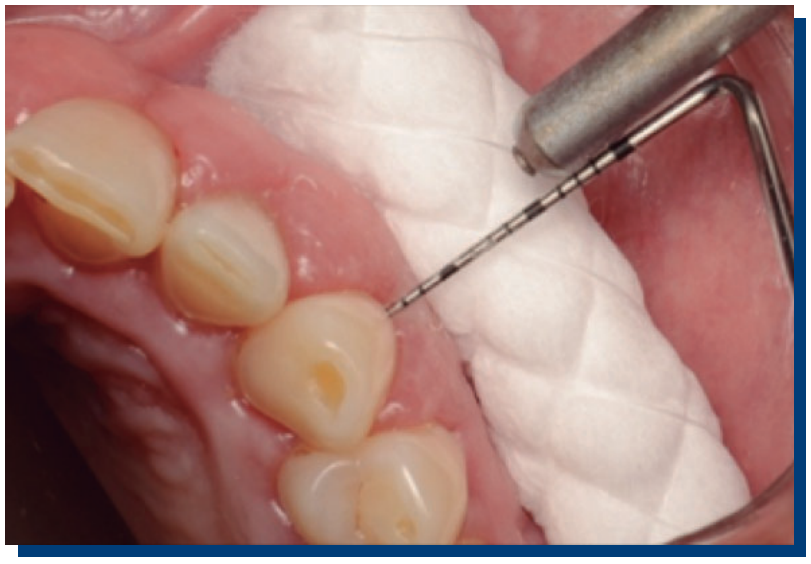

Figura 2. Posición perpendicular de la sonda periodontal a la superficie cervical vestibular. Distancia de la jeringa triple a $1 \mathrm{~cm}$ de la pieza dentaria.

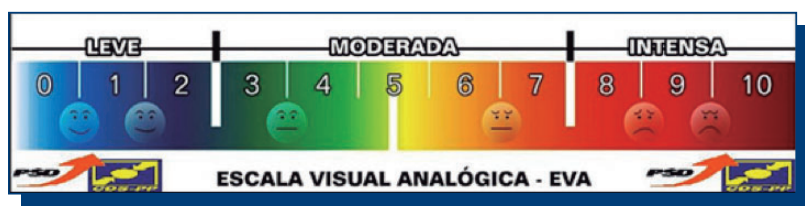

Figura 3. Escala visual análoga.

Tabla 1. Valores promedios y desviación estándar del EVA inicial, y durante la primera y tercera semana del dolor.

\begin{tabular}{|c|c|c|c|}
\hline Grupo & $\begin{array}{c}\text { EVA Inicial } \\
\text { X+- DS }\end{array}$ & $\begin{array}{c}\text { EVA Primera } \\
\text { semana } \\
\text { X+- DS }\end{array}$ & $\begin{array}{c}\text { EVA Tercera } \\
\text { semana } \\
\text { X+- DS }\end{array}$ \\
\hline Colgate & $6.5+-1.179$ & $4.5+-1.354$ & $3.5+-1.434$ \\
\hline Sensodyne & $7.1+-1.287$ & $5.2+-1.398$ & $3.8+-1.764$ \\
\hline
\end{tabular}

Tabla 2. Intervalo de Confianza del EVA inicial, y durante la primera y tercera semana del dolor.

\begin{tabular}{|c|c|c|c|}
\hline Grupo & $\begin{array}{c}\text { EVA Inicial } \\
\text { IC al 95\% }\end{array}$ & $\begin{array}{c}\text { EVA Primera } \\
\text { semana } \\
\text { IC } \text { al } 95 \%\end{array}$ & $\begin{array}{c}\text { EVA Tercera } \\
\text { semana } \\
\text { IC al } 95 \%\end{array}$ \\
\hline Colgate & 5.657 a 7.343 & 3.531 a 5.469 & 2.474 a 4.526 \\
\hline Sensodyne & 6.180 a 8.020 & 4.200 a 6.200 & 2.533 a 5.245 \\
\hline
\end{tabular}

\section{RESULTADOS}

De los veinte pacientes seleccionados según los criterios de inclusión y exclusión, uno de ellos abandonó el estudio en la tercera semana de tratamiento, ya que no continuó con su tratamiento periodontal.

El promedio de edad de los pacientes fue de 53.7 años. Un $73.6 \%$ correspondió al género femenino y un $26.4 \%$ al género masculino (Tabla 3).

Durante el periodo de investigación, no se observaron o relataron efectos adversos en los tejidos duros y blandos de la cavidad oral, tanto por el examinador como por el paciente.

En el Gráfico 1 se observa que el grado de dolor medido en la escala EVA durante la primera semana de uso fue similar en ambos grupos y no presentaban diferencias estadísticamente significativas. Se observó que no hubo diferencia significativa al comparar el uso de las pastas dentales Colgate Pro Alivio ${ }^{\circledR}$ y Sensodyne Rápido Alivio®.

En el Gráfico 2 se observa que el grado de dolor medido en la escala EVA durante la tercera semana de uso fue similar en ambos grupos y no presentaban diferencias estadísticamente significativas. Se observó que no hubo diferencia significativa al comparar el uso de las pastas dentales Colgate Pro Alivio ${ }^{\circledR}$ y Sensodyne Rápido Alivio®.

Como se observa en el Gráfico 3, en ambos grupos disminuyó el dolor a la $1^{a}$ y $3^{a}$ semana, pero no presentaban diferencias entre ellas, es decir, la pendiente o disminución del dolor fue similar en ambos grupos. Sí se observó disminución significativa del dolor en ambos grupos.

Tabla 3. Resumen de edad y género.

\begin{tabular}{|c|c|c|c|c|c|}
\hline & \multicolumn{3}{|c|}{ Número de sujetos } & \multicolumn{2}{c|}{ Edad (años) } \\
\hline Tratamiento & Masculino & Femenino & Total & Promedio & Rango \\
\hline $\begin{array}{c}\text { Arginina al 8\%- } \\
\text { carbonato de } \\
\text { calcio }\end{array}$ & 3 & 7 & 10 & 50.4 & 31 a 68 \\
\hline $\begin{array}{c}\text { Acetato de } \\
\text { estroncio al 8\% }\end{array}$ & 2 & 7 & 9 & 57.5 & 48 a 67 \\
\hline Total & 5 & 14 & 19 & & \\
\hline
\end{tabular}




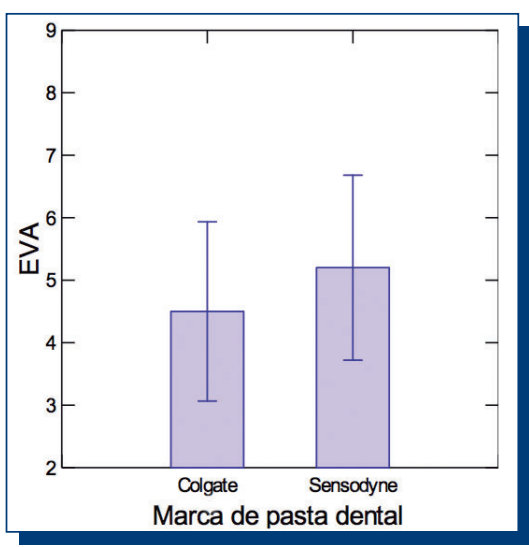

Gráfico 1. Evaluación del dolor en la primera semana de uso de los dentríicos.

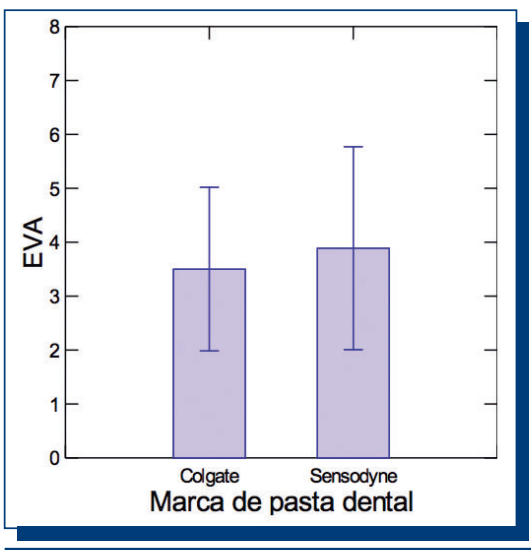

Gráfico 2. Evaluación del dolor en la tercera semana de uso de los dentríficos.

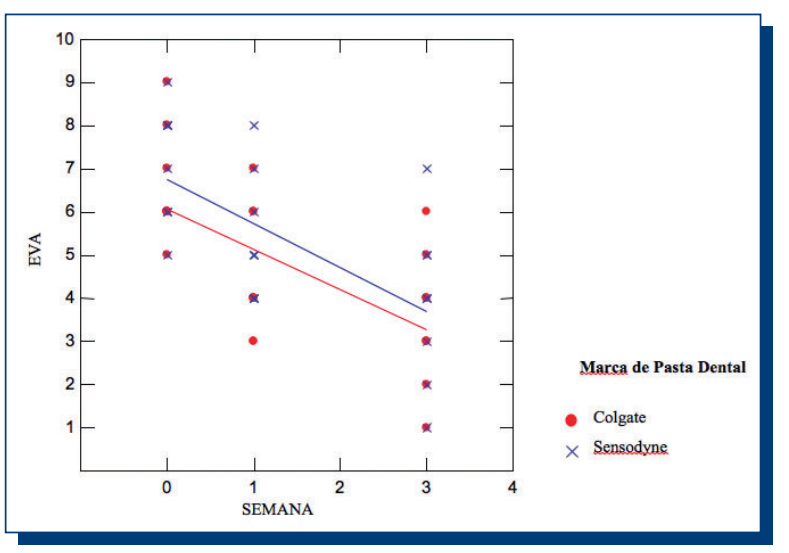

$\mathrm{Y}=7.66$ - 0.97. *Semana 0.56 Marca $r=0.66 ; r^{2}=0.44$.

Gráfico 3. Disminución del dolor según la pasta dental y semanas de uso.

\section{DISCUSIÓN}

En el presente estudio se investigó el efecto de dos pastas dentales utilizadas para el tratamiento de la hipersensibilidad dentinaria. Podemos observar que existió una disminución estadísticamente significativa de la sensibilidad con el uso de ambos dentífricos medido durante la primera y tercera semana de uso.

Al determinar la reducción de la hipersensibilidad dentinaria con el dentífrico que contiene arginina $8 \%$ - carbonato de calcio, podemos observar que hubo una disminución promedio del dolor de 3.0 unidades de EVA en las tres semanas de tratamiento. En cambio al evaluar la reducción de la hipersensibilidad dentinaria con el dentífrico que contiene acetato de estroncio al $8 \%$, podemos observar que se obtuvo una reducción de la hipersensibilidad de 3.3 unidades de EVA en las tres semanas de tratamiento. Por lo tanto, en ambos grupos la reducción del dolor fue estadísticamente significativa.

A pesar de que en este estudio se demostró que hubo una disminución de la sensibilidad con ambos agentes terapéuticos, sin mostrar diferencias significativas entre ambos, investigaciones realizadas por Márquez $\mathrm{M}$ et al., donde se comparó el efecto de la arginina $8 \%$ carbonato de calcio y del fluoruro de sodio al $5 \%$ en la reducción de la hipersensibilidad dentinaria posterior a la terapia periodontal, se observó una mayor eficacia en la reducción del dolor en el grupo que utilizó arginina al $8 \%$ y carbonato de calcio en comparación con la aplicación de barniz de fluoruro de sodio al $5 \%{ }^{(20)}$.

Asimismo, en estudios realizados por Schiff T et al., en el cual se evaluó clínicamente la eficacia de la pasta desensibilizante que contiene arginina $8 \%$ - carbonato de calcio en proveer un alivio instantáneo y duradero en pacientes con hipersensibilidad, se demostró que provee una reducción estadísticamente significativa en la disminución de la hipersensibilidad dentinaria después de aplicar el producto en forma inmediata, y que ésta se mantuvo por un periodo de 28 días ${ }^{(22)}$.

Por lo tanto, según los estudios mencionados anteriormente, se establece que el dentrífico que contiene arginina al $8 \%$ - carbonato de calcio, es superior a los otros agentes desensibilizantes utilizados, lo que no concuerda con los datos obtenidos en este trabajo de investigación, en el cual no hubo una diferencia estadísticamente significativa entre los dentríficos estudiados. Sin embargo, en un estudio realizado por West NX et al., se demostró que la aplicación de un dentífrico llamado NovaMin ( $5 \%$ fosfosilicato de calcio, sodio y $1.450 \mathrm{ppm}$ de flúor), fue superior al dentífrico que contiene arginina $8 \%$ - carbonato de calcio al evaluarlo en un medio ácido( ${ }^{(16)}$, lo que no coincide con las demás investigaciones citadas previamente. Por ende, podríamos concluir que la efectividad de los dentífricos desensibilizantes podrían ser influenciados por variantes biológicas, como la saliva, ingesta excesiva de ácidos y/o el comportamiento del paciente.

Por otro lado, la investigación realizada por Gilliam et al., se comparó la eficacia de tres pastas dentales. La primera contenía 3.75\% de cloruro de potasio, la segunda contenía $8 \%$ de acetato de estroncio y la tercera correspondía al grupo control. Después de seis semanas de aplicación del producto, hubo una reducción estadísticamente significativa de la sensibilidad en los tres grupos, pero no hubo diferencia significativa entre los productos aplicados ${ }^{(23)}$.

Por lo tanto, el estudio previamente mencionado respalda que los dentífricos que contienen acetato de estroncio al $8 \%$ no fueron superiores al resto de los agentes terapéuticos utilizados, lo que coincide con los resultados obtenidos en este estudio, ya que no se observaron diferencias estadísticamente significativas entre la pasta dental que contiene arginina al $8 \%$ - carbonato de calcio versus el dentífrico que contiene acetato de estroncio al $8 \%$.

Al evaluar el Gráfico 3 podemos observar que en ambos grupos disminuyeron los niveles de sensibilidad, y que durante la tercera semana de tratamiento fue cuando más disminuyó, pero no hubo una diferencia significativa entre los agentes desensibilizantes utilizados.

No obstante, estos resultados no concuerdan con publicaciones previas, ya que según estudios realizados por Schiff y col. en relación a la comparación entre los dentífricos que contienen arginina $8.0 \%$, carbonato de calcio y 1.450 ppm de flúor (Colgate Pro Alivio $($ )), y acetato de estroncio $8 \%$ con 1.040 ppm de flúor (Sensodyne Rápido Alivio $\left.{ }^{\circledR}\right)$, respaldan la conclusión que al cepillarse durante ocho semanas con la pasta Colgate Pro Alivio®, provee una reducción significativa de la hipersensibilidad dentinaria comparada con el uso del dentífrico Sensodyne Rápido Alivio ${ }$, aplicada del mismo modo. Además, establece que la reducción de la hipersensibilidad dentinaria lograda con el uso del dentífrico Sensodyne Rápido Alivioß, aplicada dos veces al día, es significativamente mejorada al cambiar el dentífrico por Colgate Pro Alivio®; y que la reducción de la hipersensibilidad dentinaria lograda con la pasta dental Colgate Pro Alivio ${ }^{\circ}$, aplicada dos veces al día, no es mejorada al cambiar el dentífrico por Sensodyne Rápido Alivio ${ }^{\circledR}$, aplicado durante dos veces al día por dos u ocho semanas. Finalmente, se demostró que el uso durante ocho semanas con la pasta Colgate Pro Alivio $\AA$, tras el uso con Sensodyne Rápido Alivio $₫$, durante el mismo periodo, provee una reducción significativa de la hipersensibilidad dentinaria en relación con el uso de Sensodyne Rápido Alivio ${ }$, aplicada durante el mismo tiempo ${ }^{(24)}$. 
Sin embargo, en este trabajo de investigación, no se observó una diferencia estadísticamente significativa entre la aplicación de ambos agentes terapéuticos.

\section{CONCLUSIÓN}

No existen diferencias estadísticamente significativas entre el uso de los dentífricos que contienen arginina 8\% - carbonato de calcio y acetato de estroncio al $8 \%$ para la reducción de la hipersensibilidad dentinaria tras una y tres semanas de uso de las pastas dentales.

Tanto los dentífricos que contienen arginina $8 \%$ - carbonato de calcio como la que contiene acetato de estroncio al $8 \%$, fueron efectivos en la reducción de la hipersensibilidad dentinaria tras una y tres semanas de uso, logrando una diferencia estadísticamente significativa en la reducción del dolor con ambas pastas.

No existe una diferencia estadísticamente significativa entre ambos agentes desensibilizantes, en relación a la diferencia en la disminución de la sensibilidad desde la primera hasta la tercera semana de aplicación.

A pesar de que los resultados son positivos en relación a la disminución de la sensibilidad, se necesitaría obtener un mayor tamaño de la muestra, y además prolongar el tiempo de uso del agente terapéutico para hacerlos comparables, ya que en la mayor parte de las experiencias los agentes son utilizados en periodos de seis semanas.

Una de las limitaciones que podría haber influenciado en el presente estudio, es que no fue posible tener certeza del cumplimiento de las indicaciones por parte de los pacientes que utilizaron los dentífricos.

También, podríamos decir que al entregarles las pastas dentales a los pacientes, pudo haber existido sesgo por alguna información obtenida por el paciente, sugestionando de este modo al sujeto.

En este trabajo de investigación, se refirió al dolor experimentado después del tratamiento periodontal medido en una Escala Visual Análoga. Esto fue difícil de cuantificar, porque la percepción del dolor ante un estímulo similar es muy variable entre los individuos.

Otra limitación fue el abandono de este estudio por parte de un paciente en la tercera semana de tratamiento, lo que pudo haber influenciado los resultados estadísticos al disminuir el número de la muestra.

Se sugiere en investigaciones futuras que se considere un tiempo mayor de tratamiento con cada pasta dental para observar si hay diferencias estadísticamente significativas entre los dentífricos que contienen arginina $8 \%$ - carbonato de calcio y la que contiene acetato de estroncio al $8 \%$ a través del tiempo. Realizar un estudio tipo doble ciego y longitudinal con el objetivo de que los pacientes desconozcan el tipo de tratamiento utilizado, disminuyendo la posibilidad de que existan sesgos, para poder lograr una mayor precisión de los resultados. Además, se sugiere realizar un seguimiento a los pacientes, para poder tener certeza del efecto de los agentes a largo plazo y que se evalúen otras variables como el cumplimiento del tratamiento, de las indicaciones dadas a los pacientes y de los efectos adversos en la cavidad oral.

\section{CONFLICTO DE INTERESES}

Los autores declaran no tener conflictos de interés.

\section{REFERENCIAS BIBLIOGRÁFICAS}

1. Carranza F, Sznajder N. Clasificación de las enfermedades periodontales. Compendio de Periodoncia, $5^{\circ}$ ed. Buenos Aires: Editorial Médica Panamericana 1996: 15.

2. Carranza F, Newman M. Principios generales de la cirugía periodontal. Periodontología Clínica, $9^{\circ}$ ed. Barcelona: McGraw-Hill; 2002: 733

3. Ten Cate AR. Histología oral: Desarrollo, estructura y función. Periodonto, $2^{\circ}$ ed. Buenos Aires: Editorial Médica Panamericana; 1986: 291

4. Chu C. Management of dentine hypersensitivity. Dent Bull, 2010; 15: 21-23.

5. Chu C, Lo E. Dentine hypersensitivity: A review. Hong Kong Dent J, 2010; 7: $15-22$.

6. Cummins D. Recent advances in dentin hypersensitivity: Clinically proven treatments for instant and lasting sensitivity relief. Am J Dent, 2010; 23(Spec Iss): $3 \mathrm{~A}-13 \mathrm{~A}$.

7. Kielbassa A. Dentine hypersensitivity: Simple steps for everyday diagnosis and management. Int Dent J, 2000; 52: 394-396.

8. Kuchenbecker C. Dentine hypersensitivity: Analysis of self-care products. Braz Oral Res, 2009; 23(Spec Iss): 53-63.

9. Orchardson R, Gillam D. Managing dentine hypersensitivity. J Am Dent Assoc, 2006; 137: 990-998.

10. Panagakos F, Schiff T, Guignon A. Dentine hypersensitivity: Effective treatment with an in-office desensitizing paste containing $8 \%$ arginine and calcium carbonate. Am J Dent, 2009; 22(Spec Iss): 3-7.

11. Porto I, Andrade A, Montes M. Diagnosis and treatment of dentina hypersensitivity. J Oral Sci, 2009; 51: 323-332.

12. Walters P. Dentinal hypersensitivity: A review. J Contemp Dent Pract, 2005; 6: 107-117.

13. Wara-aswapati N, Krongnawakul D, Jiraviboon D, Adulyanon D, Karimbyx N, Pitiphat W. The effect of a new toothpaste containing potassium nitrate and triclosan on gingival health, plaque formation and dentine hypersensitivity. J Clin Periodontol, 2005; 32: 32-53.

14. West NX. Dentine hypersensitivity: Preventive and therapeutic approaches to treatment. Periodontology 2000, 2008; 48: 31-41.
15. West NX. The dentine hypersensitivity patient-a total management package. Int Dent J, 2007; 57: 411-419.

16. West NX. Randomized in situ clinical study comparing the ability of two new desensitizing toothpaste technologies to occlude patent dentin tubules. J Clin Dent 2011; 22(Spec Iss): 82-89.

17. Pol D, Jonnala J, Chute M, Gunjikar T, Pol S. The role of oral higiene and periodontal condition/disease in aetiology, prevention and management of dentine hypersensitivity. J Indian Dent Assoc, 2011; 5: 664-666.

18. Cuniberti N, Rossi G. Lesiones cervicales no cariosas: La lesión dental de futuro. Editorial Médica Panamericana,Buenos Aires; 2009. p.121.

19. Lavender S, Petrou I, Heu R. Mode of action: Studies on a new desensitizing dentifrice containing $8.0 \%$ arginine, a high cleaning calcium carbonate system and 1.450 ppm fluoride. Am J Dent, 2010; 23(Spec Iss): 14-19.

20. Márquez M, Quintero A, Sanz A, Ramírez V, Inostroza C, Chaparro A. Efecto de la arginina $8 \%$ - carbonato de calcio y del fluoruro de sodio al $5 \%$ en la reducción de la hipersensibilidad dentinaria post terapia periodontal: Ensayo clínico. Rev Clin Periodoncia Implantol Rehabil Oral, 2011; 4: 22-25.

21. Parkinson CR, Willson RJ. A comparative in vitro study investigating the occlusion and mineralization properties of commercial toothpastes in a four-day dentin disc model. J Clin Dent, 2011; 22(Spec Iss): 74-81.

22. Schiff T, Delgado E, Zhang Y, Cummins D, De Vizzio W, Mateo L. Clinical evaluation of the efficacy of an in-office desensitizing paste containing $8 \%$ arginine and calcium carbonate in providing instant and lasting relief hypersensitivity. $A m J$ Dent, 2009; 22(Spec Iss): 8-15.

23. Gilliam DG, Bulman JS, Jackson R, Bullman HN. Comparision of two desensitizing dentifrices with a commercially available fluoride dentifrice in alleviating cervical dentine hypersensitivity. J Periodontol, 1996; 67: 737-742.

24. Schiff T, Mateo LR, Delgado E, Cummins D, Zhang YP, De Vizio W. Clinical efficacy in reducing dentin hypersensitivity of a dentifrice containing $8.0 \%$ arginine calcium carbonate, and $1.450 \mathrm{ppm}$ fluoride compared to a dentrifice containing $8 \%$ strontium acetate and $1.040 \mathrm{ppm}$ fluoride under consumer usage before and after switch-over. J Clin Dent, 2011; 22(Spec Iss): 128-138. 